\title{
A ROBUST INVARIANCE APPROACH TO IDLE SPEED CONTROL OF A SPARK IGNITED ENGINE ${ }^{1}$
}

\author{
Paolo Caravani, Giovanni Girasole ${ }^{2}$ \\ Electrical Engineering Department, University of L'Aquila, \\ Monteluco di Roio, 67040 L'Aquila,Italy \\ caravani@ing.univaq.it, girasole@ing.univaq.it
}

\begin{abstract}
Idle speed control of a spark ignition engine is studied via a control invariant approach. A hybrid system describes torque generation at cycle-level. The continuous-time dynamics is reset at prescribed crankshaft angles. Timediscretization allows to handle delays in the control loop and constraints on state and control variables in a simple form by means of a control invariant technique. The synchronization problem is solved by a robust extension of the same technique. Controller implements a piecewise-linear state-feedback law. Copyright ${ }^{\circledR} 2005$ IFAC.
\end{abstract}

Keywords: Robust Control, Constrained Systems, Control Invariance, Box Invariance

\section{INTRODUCTION}

Three main features are shared by idle speed control (ISC) of spark ignition engines: nonlinearities, delays in the control loop and \& synchronization, model and disturbance uncertainty. Control objectives typically include good tracking properties, disturbance rejection, robustness. In idle speed mode motor torque is controlled essentially by two variables, the throttle valve opening angle regulating the amount of air intake into the cylinders, and the spark ignition advance. Given the narrow range in which variables must be kept, linearized models in ISC are generally accepted and widely used in practice [Hrovat et al., 1997, Yurkovi et al., 1997]. The open-loop behaviour can be described in the time-domain or in the crankshaft-angle domain [Chin et al., 1986, Yurkovi et al., 1997].

\footnotetext{
1 The work has been conducted with partial support by MIUR under PRIN02 and IST-2001-38314 COLUMBUS Project.

2 The authors would like to thank PARADES researchers for their support and useful suggestions.
}

Mathematically, these are equivalent descriptions, the two domains being related by

$$
\frac{d x}{d t}=\frac{d x}{d \theta_{c}} \frac{d \theta_{c}}{d t}=K_{c} \dot{n} \frac{d x}{d \theta_{c}}
$$

with $\theta_{c}$ crankshaft angle and $n$ angular velocity. Since crankshaft angular velocity is a natural state variable, any model variable $x$ undergoes a non-linear transformation going from the $t$ to the $\theta_{c}$ domain. Newton's law for instance, $\frac{d \dot{n}}{d t}=$ $T$, takes on the non-linear form $\frac{d \dot{n}}{d \theta_{c}}=\frac{1}{\dot{n} K_{c}} T$ in $\theta_{c}$ domain. The advantage of the $t$-domain is that of retaining a linear description. This is a remarkable advantage since control techniques handling hard constraints, disturbance rejection and model uncertainty - control invariant techniques - are much more developed in the linear case. In the linear discrete-time case, invariant set computations are further simplified and, as linear processing of signals can be done at quite fast a rate by modern digital technology, it becomes possible to keep track of variables at engine-cycle level. However, control signal processing requires 
sampling at a fixed rate and a time-discretization somewhere in the plant-controller loop is necessary. This introduces synchronization difficulties since the motor torque is physically exerted only in a neighborhood of the engine top dead center angle. These difficulties in principle disappear in the $\theta_{c}$-domain, but re-appear under different form as soon as the non-linear domain transformation is linearized about a nominal angular speed - a hardly avoidable step if more sophisticated control design techniques like [Abate et al., 1994] or [Mayne, 2001] are to be used. More recently, approaches based on hybrid systems have been proposed [Balluchi et al., 2000] to describe engine dynamics at cycle-level as a set of separate dynamic systems evolving in continuous-time with discrete-time switches and variable resets driven by internal (engine-cycle) or external (torque disturbance) events. Our perspective in this paper is similar. We model the engine as a continuous-time dynamic system whose state is reset at prescribed - and observed - crankshaft angles. This allows to describe torque generation and control at cyclelevel. As motor torque is updated at each topdead center angle, and this is a measurable event, the engine is viewed as a hybrid system with event-driven state resets. When continuous-time dynamics is discretized for control implementation purposes, a synchronization problem arises as the new state reset occurs after a time $\delta t$ from the old, variable with engine speed. However, if speed is comprised between a lower and an upper bound (as ISC specs demand) state reset can only occur after a delay from the last event comprised between a maximum and a minimum. In Sec. 2 we describe the model and, in Sec. 3 its discretized version. In Secs. 4 we briefly outline the methodology employed. This is based on a novel approach to compute polyhedral invariants. We first impose invariance every $N$ steps of the discrete time dynamics. Our interest towards the $N$-step dynamics lies in the fact that, under reachability assumptions, the extended control matrix $B$ is full-rank and this avoids notorious difficulties associated with the computation of an invariant set $S_{N}$ in state space. We estimate in Sec. 5 a second set $S$ such that trajectories starting in $S_{N}$ stay in $S$ and in fact select a control law minimizing departure from $S_{N}$ of trajectories originating in it. Some simulated results and conclusions are reported in Sec. 6.

\section{IDLE SPEED ENGINE MODEL}

The hybrid engine model considered in this paper comprehends a continuous time component and an event-driven reset function. The continuoustime component is

$$
\begin{aligned}
& \dot{p}=a_{p} p+b_{p} \alpha \\
& \dot{n}=a_{n} n+a_{n p} p+b_{n}\left(T-T_{l}\right) .
\end{aligned}
$$

The first equation describes pressure dynamics in the intake manifold in terms of the throttle opening angle $\alpha$. Pressure dynamics depends nonlinearly on crankshaft speed. However, speed effects are negligible in idle mode and a linear relationship for $\dot{p}$ is justified. The second equation describes crankshaft dynamics in terms of angular velocity $n$. The term $a_{n} n$ accounts for dynamic friction of rotational mechanisms in the driveline. The term $a_{n p} p$ is pumping friction due to air-fuel mixture transport, opposing crankshaft rotation. The external load torque $T_{l}$ is regarded as a disturbance. The variable $T$ is a proxy of the instanteneous motor torque; in fact the real torque contributed by a single cylinder as a function of $\theta_{c}\left(\dot{\theta}_{c}=K_{c} n\right)$ exhibits a sharp positive peak immediately after the spark, and small negative values elsewhere as shown in Figure (1). $T$ is an equivalent ${ }^{3}$ torque assumed positive and constant throughout the expansion stroke, and zero elsewhere.

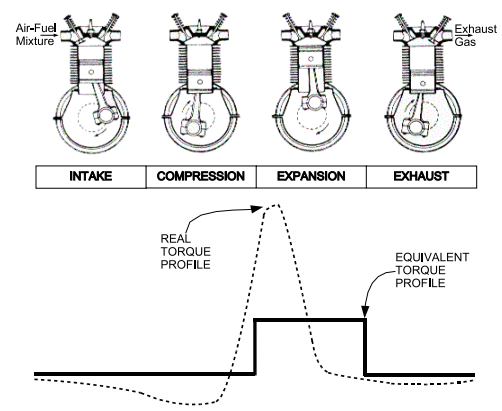

Fig. 1. Torque generation for a single cylinder

In a multi-cylinder engine there is torque overlap. In a 4 -cylinder 4 -stroke engine ${ }^{4}$ the overall torque profile is shown in Figure (2). In this case cylinders change stroke at the same time (every 180 degrees of the crankshaft angle) and there is only one cylinder active in each stroke. We say that the $e n$ -

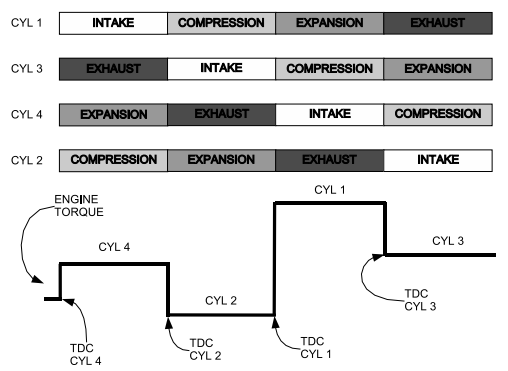

Fig. 2. Torque generation for a 4-cylinder engine

\footnotetext{
3 in terms of an energy balance.

4 in what follows we refer to this case but, the situation is similar in other engine configurations
} 
gine is at a top dead center (TDC) when one of the cylinders is at a compression TDC. Combining the equivalent torques of each cylinder, the resulting profile will be a piecewise constant function with breakpoints 180 degrees apart.

Torque is a function of the manifold pressure $p^{-}$ and of the spark advance $\theta_{s}^{-}$decided at the last engine TDC. It can be assumed

$$
T=c_{1} p^{-}+c_{2} \theta_{s}^{-}+c_{3},
$$

where $p^{-}, \theta_{s}^{-}$are values of air pressure and spark advance at the end of intake stroke.

The range in which the linear model is meaningful is described by upper and lower bounds on each of the variables $p, T, \alpha, \theta_{s}$ and $T_{l}$. The control objective is to keep engine speed in the range

$$
n_{\min } \leq n \leq n_{\max }
$$

for all possible values of the load torque $T_{l}$ in its range. Assuming engine to be at a TDC at time $t=0$, the dynamics for $0 \leq t<t_{r}$, where $t_{r}$ is the time of the next TDC when torque is reset, is

$$
\dot{x}=A_{c} x+B_{c} \alpha+C_{c} T_{l}
$$

where $x=\left\{\begin{array}{lll}p & n & T\end{array}\right\}^{\prime}$. At $t=t_{r}$ torque is reset on the basis of prior values $p^{-}, \theta_{s}^{-}$. We assume the following

A1 The angular position of the crankshaft is known at engine TDCs

Assumption 1 captures the fact that the attainment of a TDC is a measurable event that can be profitably exploited in the controller synthesis.

The actual control algorithm is implemented in the electronic control unit (ECU) which is a digital system. This feature suggests to handle delays and controller design via a numerical control approach.

\section{DISCRETIZATION AND $N$-STEP DYNAMICS}

Consider the discrete-time version of the model. The time between two engine TDCs is subdivided into $N-1$ equal intervals of length $\delta t$ and an $N$-th interval of length $\leq \delta t$.

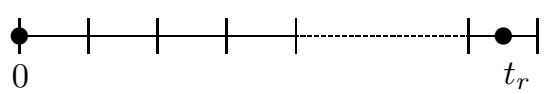

Fig. 3. Engine TDC at $t=0$ and at $t_{r}$, between $(N-1) \delta t$ and $N \delta t$

The discrete-time evolution within one engine cycle is

$$
x(k+1)=A_{d} x(k)+B_{d} \alpha(k)+C_{d} T_{l}(k)
$$

if $k=0,1 \ldots N-2$,

$$
\begin{aligned}
x^{-}(N)=A_{\tau} x(N-1) & +B_{\tau} \alpha(N-1)+ \\
& +C_{\tau} T_{l}(N-1)
\end{aligned}
$$

before reset, and

$$
x(N)=\bar{A}_{1} x(0)+\bar{B} \theta_{s}(0)+\bar{C} c_{3}+\bar{A}_{2} x^{-}(N)(6)
$$

after reset, where subscript $d$ denotes discretization with a fixed sample time $\delta t$, subscript $\tau$ discretization with a variable sample time $0 \leq \tau \leq \delta t$ and $\bar{A}_{1,2}, \bar{B}, \bar{C}$ are reset matrices. In the third equation notice the delayed effect of pressure and spark advance, as per eq. (2).

\section{N-STEP BOX INVARIANCE}

We are interested in the $N$-step dynamics, e.g. the evolution of state control and disturbance variables every $N$ time-steps. Thus labelling

$t_{k}$ the time-instant $k \delta t$

$t_{k}+1$ the time-instant $(k+N) \delta t$

$t_{k}+2$ the time-instant $(k+2 N) \delta t$

the discrete-time description of the $N$-step dynamics is

$$
x\left(t_{k}+1\right)=A x\left(t_{k}\right)+B u\left(t_{k}\right)+C v\left(t_{k}\right)
$$

where $t_{k} \in I$, the set of non-negative integers, $x \in \mathbb{R}^{3}$ and $u, v \in \mathbb{R}^{(N+1)}$ with

$$
\begin{gathered}
u\left(t_{k}\right)=\left[\begin{array}{ll}
\theta_{s}(k) & \alpha(k) \ldots \alpha(k+N-1)
\end{array}\right]^{\prime} \\
v\left(t_{k}\right)=\left[\begin{array}{ll}
c_{3} & T_{l}(k) \ldots T_{l}(k+N-1)
\end{array}\right]^{\prime}
\end{gathered}
$$

It is easily checked that matrix $B$ is full rank. Notice if a feedback law $u=F(x)$ is found, this means that the state $x\left(t_{k}\right)$ at time $k \delta t$ determines the control $\theta_{s}(k \delta t)$, plus the $N$ controls $\alpha(k \delta t) \ldots \alpha((k+N-1) \delta t)$. The set of all these controls determines the state at time $(k+$ $N) \delta t$ which is $x\left(t_{k}+1\right)$. In other words, the system evolves closed loop for $t$ in the set $\{(k+$ $m N) \delta t, \quad m=0,1, \ldots\}$ and open loop for $t$ not in this set. State constraints can be represented as a box

$$
X=\{x: \underline{x} \leq x \leq \bar{x}\}
$$

where $\underline{x}_{1}=p_{\min }, \quad \bar{x}_{1}=p_{\max }, \quad \underline{x}_{2}=n_{\min }, \quad \bar{x}_{2}=$ $n_{\max }, \quad \underline{x}_{3}=0$ and $\bar{x}_{3}=T_{\max }$.

Disturbance constraints are represented by a box 


$$
V=\{v: \underline{v} \leq v \leq \bar{v}\}
$$

where $\underline{v}_{1}=\bar{v}_{1}=c_{3}$, and $\underline{v}_{i}=0, \bar{v}_{i}=$ $T_{\text {lmax }}$ for $i>1$; disturbance $v$ is admissible if it belongs to $V$.

Control constraints are represented by a box

$$
U=\{u: \underline{u} \leq u \leq \bar{u}\}
$$

where $\underline{u}_{1}=\theta_{\min }, \bar{u}_{1}=\theta_{\max }$, and $\underline{u}_{i}=\alpha_{\min }, \bar{u}_{i}=$ $\alpha_{\max }$ for $i>1$; control $u$ is feasible if it belongs to $U$.

Let $\mathcal{R}(X)$ be the reach set of $X$, e.g. the set of states from which system (7) can reach $X$ in one step with a feasible control for all admissible disturbances

$$
\begin{array}{r}
\mathcal{R}(X)=\{x: \exists u \in U: A x+B u+C v \in X \\
\forall v \in V\}
\end{array}
$$

As is well known - and easy to check - invariance of $X$ holds if and only if

$$
X \subset \mathcal{R}(X) .
$$

A set satisfying (13) has the property that once the system state is in it, it is possible to keep it there with a feasible control for any admissible (and unknown) disturbance. In partucular, for constant admissible disturbance $X$ must contain the equilibrium point of (7). Set now

$$
B u=\sigma
$$

and assume $\sigma \in Q$ where

$$
Q=\{\sigma: \underline{\sigma} \leq \sigma \leq \bar{\sigma} .\}
$$

In terms of the new control $\sigma$ the discrete-time $N$-step dynamics becomes

$$
x\left(t_{k}+1\right)=A x\left(t_{k}\right)+\sigma\left(t_{k}\right)+C v\left(t_{k}\right) .
$$

We first establish conditions on $\underline{\sigma}, \bar{\sigma}$ ensuring invariance of $X$ for system (16). Subsequently we use these to infer bounds on $u$ for system (7).

Notation: given matrix $A=\left[a_{i j}\right]$, we denote by $A_{i}$ its $i$-th row, $A^{+}=\left[\max \left(a_{i j}, 0\right)\right], A^{-}=$ $\left[\min \left(a_{i j}, 0\right)\right]$ (and $A=A^{+}+A^{-}$with $A^{+} \geq$ $\left.0, A^{-} \leq 0\right)$. Given $p$ matrices $A^{1} \ldots A^{p}$, their convex hull is $\operatorname{Conv}\left(A^{1} \ldots A^{p}\right)=\cup_{\alpha \in S} \sum_{i} \alpha_{i} A^{i}$ where $S$ is the unit simplex of $R^{p}$.

Theorem 1. i. Set $X$ is invariant for (16) under $(10,15)$ if and only if

$$
\left[\begin{array}{cc}
A^{+}-I & A^{-} \\
-A^{-} & -A^{+}+I \\
-I & I
\end{array}\right]\left[\begin{array}{l}
\bar{x} \\
\underline{x}
\end{array}\right] \leq\left[\begin{array}{r}
-\bar{w}-\underline{\sigma} \\
\underline{w}+\bar{\sigma} \\
\underline{w}-\bar{w}
\end{array}\right]
$$

where

$$
\begin{aligned}
& \underline{w}_{i}=\min _{\underline{v} \leq v \leq \bar{v}} C_{i} v=C_{i}^{+} \underline{v}+C_{i}^{-} \bar{v} \\
& \bar{w}_{i}=\max _{\underline{v} \leq v \leq \bar{v}} C_{i} v=C_{i}^{+} \bar{v}+C_{i}^{-} \underline{v}
\end{aligned}
$$

ii. Under this condition invariance is achieved by controls satisfying $\max (\underline{\sigma}, \underline{x}-\underline{w}-A x) \leq \sigma \leq \min (\bar{\sigma}, \bar{x}-\bar{w}-A x)$

with max, min taken componentwise.

Proof We first calculate $\mathcal{R}(X)$ for system (16)

$$
\begin{gathered}
\mathcal{R}(X)=\{x: \exists \sigma \in Q: \underline{x} \leq A x+\sigma+C v \leq \bar{x}, \forall v \in V\} \\
=\{x: \exists \sigma \in Q: \underline{x}-\underline{w} \leq A x+\sigma \leq \bar{x}-\bar{w}\}(20)
\end{gathered}
$$

with $\underline{w}, \bar{w}$ as defined in the statement. Notice that

$$
\mathcal{R}(X) \neq \emptyset \quad \Rightarrow \quad \bar{w}-\underline{w} \leq \bar{x}-\underline{x}
$$

which is the last row of the matrix inequality in $i$. Assuming this holds

$$
\begin{array}{r}
\mathcal{R}(X)=\{x: \exists \sigma \in Q: \underline{x}-\underline{w}-A x \leq \\
\leq \sigma \leq \bar{x}-\bar{w}-A x\}
\end{array}
$$

$=\{\{x: \underline{x}-\underline{w}-A x \not{E} \bar{\sigma}\} \cup\{x: \underline{\sigma} \not \leq \bar{x}-\bar{w}-A x\}\}^{c}$

where $c$ denotes set complement. By De Morgan's law $\{\mathcal{A} \cup \mathcal{B}\}^{c}=\mathcal{A}^{c} \cap \mathcal{B}^{c}$ we obtain

$$
\begin{aligned}
\mathcal{R}(X)= & \{x: \underline{x}-\underline{w}-A x \leq \bar{\sigma}\} \cap \\
& \cap\{x: \underline{\sigma} \leq \bar{x}-\bar{w}-A x\}= \\
= & \{x: \underline{x}-\underline{w}-\bar{\sigma} \leq A x \leq \bar{x}-\bar{w}-\underline{\sigma}\}
\end{aligned}
$$

Next, we impose invariance

$$
X \subset \mathcal{R}(X) \Leftrightarrow\left\{\begin{array}{l}
\max \quad A_{i} x \leq[\bar{x}-\bar{w}-\underline{\sigma}]_{i} \\
\max -A_{i} x \leq[-\underline{x}+\underline{w}+\bar{\sigma}]_{i} \\
\underline{x} \leq x \leq \bar{x} \quad i=1 \ldots n
\end{array}\right.
$$

or

$$
\begin{gathered}
A^{+} \bar{x}+A^{-} \underline{x} \leq \bar{x}-\bar{w}-\underline{\sigma} \\
-A^{-} \bar{x}-A^{+} \underline{x} \leq-\underline{x}+\underline{w}+\bar{\sigma}
\end{gathered}
$$

Inequalities $(25,26)$ are the first two rows of the matrix inequality in $i$. Under condition $i$. there exists a feasible $\sigma$ satisfying the inequality in (22), which proves $i$.

We remark that if the origin is contained in the interior of $X$ and $V$ and if $\min _{i} \quad \bar{w}_{i}-\max _{i} \underline{w}_{i}>0$, then inclusion (13) in absence of noise implies contractivity as well as invariance of $X$, e.g. there exists $\lambda \in\left(\begin{array}{ll}0 & 1\end{array}\right)$ such that under a feasible control $X$ maps into $\lambda X$ at each step. This ensures asymptotic stabilizability of the equilibrium point. Notice also that from the inequalities in $i i$. the choice of $\sigma$ given $x$ is not unique. For instance, a possible control law is the midpoint control law, where $\sigma$ is chosen as the arithmetic mean of its bounds.

Theorem 2. i. Set $X$ is invariant for (7) under $(10,11)$ if

$$
\{u: \underline{\sigma} \leq B u \leq \bar{\sigma}\} \subset\{u: \underline{u} \leq u \leq \bar{u}\}
$$

with $\bar{w}, \underline{w}, \underline{\sigma}, \bar{\sigma}$ satisfying (17) is non-void. 
ii. Under this condition invariance is achieved by controls satisfying

$$
\begin{aligned}
\max (\underline{\sigma}, \underline{x}-\underline{w}-A x) \leq B u \\
\leq \min (\bar{\sigma}, \bar{x}-\bar{w}-A x)
\end{aligned}
$$

\section{Proof Directly from Thm 1.}

Notice that the bounds for $B u$ are piecewise linear-affine functions of $x$. This makes the control law simple to implement. Notice also that these bounds do not determine $u$ uniquely. Such a degree of freedom can be usefully exploited to limit, e.g. minimize, departure of the state from $X$ in the time intervals comprised between two consecutive TDCs. Before undertaking this minimization, we establish the following robustness result.

\section{Theorem 3. Given $(7,9,10)$}

i. If the conditions of Thm 1 are satisfied for $A^{i}, B^{i}, C^{i}$ with $\underline{w}^{i}, \bar{w}^{i}, \underline{\sigma}^{i}, \bar{\sigma}^{i} \quad i=1 \ldots p$ then $X$ is invariant for any system with

$$
A, B, C \in \operatorname{Conv}\left(A^{1}, B^{1}, C^{1} \ldots A^{p}, B^{p}, C^{p}\right) .
$$

ii. The control law satisfying inequalities (27) with

$$
\begin{aligned}
\underline{w} & =\min _{i} \underline{w}^{i}, & \bar{w} & =\max _{i} \bar{w}^{i} \\
\underline{\sigma} & =\min _{i} \underline{\sigma}^{i}, & \bar{\sigma} & =\max _{i} \bar{\sigma}^{i}
\end{aligned}
$$

and $A, B$ replaced by $A^{i}, B^{i}, i=1 \ldots p$ makes $X$ invariant for any of the above systems.

Proof Let $\mathcal{R}_{i}(X)$ be the reach set of $X$ under system $\left(A^{i}, B^{i}, C^{i}\right)$ and $\mathcal{R}_{\alpha}(X)$ the same under $\sum \alpha_{i}\left(A^{i}, B^{i}, C^{i}\right)$ (with $\alpha$ in the unit simplex of $\left.\mathbb{R}^{p}\right)$. If conditions of Thm 1 hold, $\cap_{i} \mathcal{R}_{i}(X)$ is non-void and satisfies $X \subset \cap_{i} \mathcal{R}_{i}(X)$. Due to convexity, $\cap_{i} \mathcal{R}_{i}(X) \subset \mathcal{R}_{\alpha}(X) \forall \alpha$ and we conclude $X \subset \mathcal{R}_{\alpha}(X) \quad \forall \alpha$, which proves $i$.

Part ii. follows from the fact that if $X$ is invariant under any system with $A, B, C \in$ $\operatorname{Conv}\left(A^{1}, B^{1}, C^{1} \ldots A^{p}, B^{p}, C^{p}\right)$ with constraints $U, V$, it must keep the property with constraints $U^{\prime} \supset U, V^{\prime} \subset V$. Now if $U_{i}, V_{i}$ are the boxes defined by $\underline{w}^{i}, \bar{w}^{i}, \underline{\sigma}^{i}, \bar{\sigma}^{i}$ then $\underline{\sigma}, \bar{\sigma}$ define the minimal box containing all $U_{i}$ 's and $\underline{w}, \bar{w}$ the minimal box containing all $V_{i}$ 's.

This result permits to handle uncertanity over $\tau$; in fact, the matrices $A, B$ and $C$ are dependent on the uncertain parameter $\tau$ (see Appendix 7.1) and the $p, A^{i}, B^{i}$ and $C^{i}$ with $i=1 \ldots p$ in Thm 3 are chosen such that $(A, B, C) \in \operatorname{Conv}\left(A^{i}, B^{i}, C^{i}\right)$.

\section{INTRACYCLE DYNAMICS: OPTIMAL CHOICE OF $U$}

If $X$ is $N$-step invariant trajectories originating in $X$ return to $X$ at most every $N$ time steps.
As $X$ need not be invariant (e.g. 1-step invariant) it is of interest to estimate the smallest box that contains all trajectories originating in $X$. We term intracycle the dynamics occurring between two TDCs. Let $x_{c} \in X$. Since trajectories have finite amplitude over finite time, there exists a $\lambda<\infty$ such that if $x(0) \in X$ (see fig. 4)

$$
x(m) \in \lambda\left(X-x_{c}\right)+x_{c} \quad \forall m \in(0, N)
$$

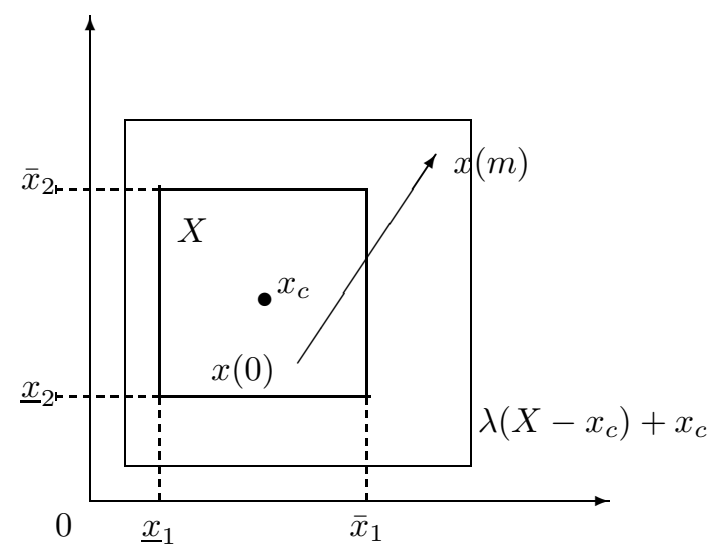

Fig. 4. Departure from $N$-step invariant box

and (29) translates into

$$
\begin{aligned}
x_{c}+\lambda\left(\underline{x}-x_{c}\right) \leq A_{m} x & +B_{m} u^{m}+C_{m} v^{m} \leq \\
& \leq x_{c}+\lambda\left(\bar{x}-x_{c}\right),
\end{aligned}
$$

with $0<m<N, u^{m}=\{u(0) \ldots u(m)\}^{\prime}$ and likewise for $v^{m}$ (intracycle matrices $A_{m}, B_{m}, C_{m}$ defined in the Appendix). As this must hold for all $v \in V$, we re-write the above as

$$
\begin{gathered}
x_{c}-\underline{w}^{m}+\lambda\left(\underline{x}-x_{c}\right) \leq A_{m} x+B_{m} u^{m} \leq \\
\leq x_{c}-\bar{w}^{m}+\lambda\left(\bar{x}-x_{c}\right) \quad m \in(0 N)
\end{gathered}
$$

where, similarly to $(18,19) \underline{w}^{m}, \bar{w}^{m}$ are given by

$$
\begin{aligned}
& \underline{w}^{m}=C_{m}^{+} \underline{v}^{m}+C_{m}^{-} \bar{v}^{m} \\
& \bar{w}^{m}=C_{m}^{+} \bar{v}^{m}+C_{m}^{-} \underline{v}^{m} .
\end{aligned}
$$

Finally, we must ensure $N$-step invariance of $X$

$$
\underline{x}-\underline{w} \leq A x+B u \leq \bar{x}-\bar{w}
$$

and satisfaction of control constraints

$$
\underline{u} \leq u \leq \bar{u}
$$

For given $x=x(0)$ we can compute $\hat{\lambda}(x)=$ $\min _{u} \lambda$ subject to $(30,31,32)-$ a LP problem. The solution to this problem yields a control law of the form

$$
x(0) \mapsto u(0) \ldots u(N-1)
$$

that guarantees $N$-step invariance of $X$, and minimal departure from $X$ of system trajectories originating in $X$. An a-priori estimate of the maximal departure from $X$ can be obtained by noting that $\hat{\lambda}-1$ is the Minkowski functional of $X$, a well known convex function yielding the bound 
$\hat{\lambda} \leq \max _{k} \hat{\lambda}\left(\xi_{k}\right)$ where $\xi_{k}$ is the $k-t h$ vertex of $X$. We conclude that $X$ is invariant for (4-6) if and only if $\max _{k} \hat{\lambda}\left(\xi_{k}\right)=1$; otherwise, $X$ is safe wrt $\left(X-x_{c}\right) \lambda+x_{c}$ for $\lambda=\max _{k} \hat{\lambda}\left(\xi_{k}\right)$.

\section{SIMULATED RESULTS AND CONCLUSION}

The graphs below illustrate the performance of the $N$-step controller on a simulated model. in correspondence of highly random changes in the load torque.

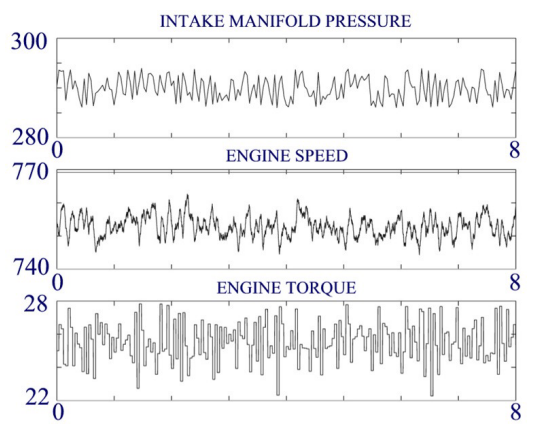

Fig. 5. Simulated result: state variables

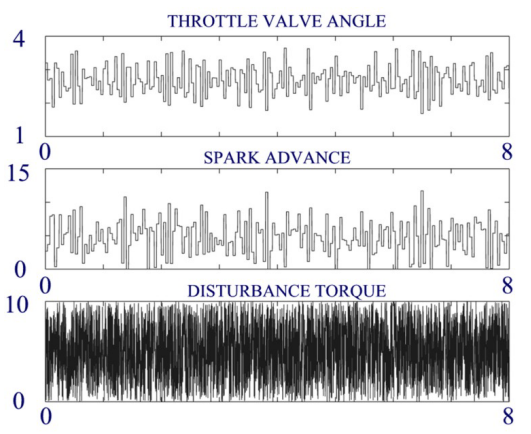

Fig. 6. Simulated result: control and disturbance variables

In conclusion, we presented an innovative technique for ISC of a spark ignition engine.

With respect to typical control tools (PID,LQ, $H^{\infty}, l^{1}$, etc) used in literature, see [Carnevale et al., 1993, Jayasuriya et al., 1994, Butts et al., 1999, Morris et al., 1982, Williams et al., 1989], our approach has the advantage of handling hard constraints on state and control variables by means of invariant techniques, see [Berardi et al., 2001]. The use of invariants of prescribed shape (boxes) while appealing in engineering practice, permits drastic simplification in the computation.

\section{APPENDIX}

\section{$7.1 N$-step dynamics}

Iterating (4) for $N-1$ steps and grouping control and disturbance terms as in (8) we get (7) with

$$
\begin{aligned}
& A=\bar{A}_{1}+\bar{A}_{2} A_{\tau} A_{d}^{N-1} \\
& B=\left[\bar{B}\left|\bar{A}_{2} A_{\tau} A_{d}^{N-2} B_{d} \ldots \bar{A}_{2} A_{\tau} B_{d}\right| \bar{A}_{2} B_{\tau}\right] \\
& C=\left[\bar{C}\left|\bar{A}_{2} A_{\tau} A_{d}^{N-2} C_{d} \ldots \bar{A}_{2} A_{\tau} C_{d}\right| \bar{A}_{2} C_{\tau}\right]
\end{aligned}
$$

\subsection{Intracycle dynamics}

$A_{m}, B_{m}, C_{m}$ are defined as in (33-35) with $N$ replaced by $m$.

\section{REFERENCES}

Abate M., Barmish R., Murillo-Sanchez C. et al., Application of some new tools to robust stability analysis of spark ignition engines: a case study, IEEE trans. on Ctrl. Sys. Technology, 2,1,1994, pp.22-30.

Balluchi A., Di Benedetto M.D., Miconi G., et al. Maximal safe set computation for idle speed control of an automated engine, in Lect. Notes in Comp. Sci. vol. 1790, Hybrid Systems: computation and control, Lynch N., Krogh B. H. Eds, Springer-Verlag 2000, pp. 32-44.

Berardi L., De Santis E., Di Benedetto, M.D. et al. Approximations of maximal controlled safe sets for hybrid systems, Proc. Worksh. on Hybr. Ctrl. and Automotive Appl. Berlin, 2001

Butts K., Sivashankar R., Jing Sun N., Application of $l^{1}$ optimal control to the engine idle speed control problem, IEEE Trans. on Ctrl. Sys. Technology, 7,2,99, pp. 258-270.

Carnevale C., Moschetti A. Idle speed control with H-infinity technique, Soc. Autom. Eng. 930770, 1993.

Chin Y., Coats F.E. Engine dynamics: time-based versus crank-angle based Soc. Autom. Eng. 860412, 1986

Hazell P. A., Flower J. O. Discrete modelling of spark ignition engines for control purposes, Int. J. of Ctrl. 13, 1971, pp. 625-632

Hrovat D., Jing Sun, Models and control methodologies for IC engine idle speed control design, Ctrl. Eng. Practice, 5,8, 97, pp. 1093-1100.

Jayasuriya S., Franchek M.A. A QFT type design methodology for a parallel structure and its applicatioin in idle speed control, Int. J. of Ctrl. 60, 5, 1994, pp. 653-670.

Mayne D.Q. Control of constrained dynamic systems, Europ. J. of Ctrl. 7,2001,pp. 87-99

Morris R. L., Warlick M. V., Borcherts R. H. Engine idle dynamics and control: a $5.8 \mathrm{~L}$ application, Soc. Auto. Eng. 820778,1982.

Williams D., Hrovat C., Davey D. et al. Idle speed control design: an H-infinity approach, Proc. Am. Ctrl. Conf. 1989, pp.1950-1956.

Yurkovi S., Simps M. Crank-angle domain modelling and control for idle speed, Soc. of Autom. Eng. 970027, 1997, pp. 34-41. 